\title{
La conservation des forêts au Népal: Encourager la participation des femmes
}

Augusta Molnar

Follow this and additional works at: https://knowledgecommons.popcouncil.org/departments_sbsr-pgy

Part of the Environmental Health and Protection Commons, Family, Life Course, and Society

Commons, Forest Sciences Commons, Gender and Sexuality Commons, International Public Health

Commons, Natural Resources and Conservation Commons, Place and Environment Commons, and the Sustainability Commons

How does access to this work benefit you? Let us know!

\section{Recommended Citation}

Molnar, Augusta. 1990. "La conservation des forêts au Népal: Encourager la participation des femmes," SEEDS no. 10. New York: Population Council. 

SEEDS est une série de brochures publiée en réponse aux demandes d'information venant du monde entier sur des idées de programmes innovatrices et pratiques, réalisées par et pour des femmes aux revenus faibles. Le but de ces brochures est de diffuser l'information et de stimuler la création de nouveaux projets, fondés sur les expériences positives de femmes qui travaillent pour améliorer leur propre statut économique et celui des autres. Les projets décrits dans ce numéro comme dans le reste de la série ont été choisis du fait qu'ils procurent aux femmes un revenu en espèces et les font participer activement aux prises de décisions comme aux profits. Ces projets sont bâtis suivant des critères économiques solides et permettent de surmonter avec succès des obstacles courants. Ces rapports n'ont cependant pas été conçus pour être suivis à la lettre car chaque effort de développement doit faire face à des ressources et des problèmes quelque peu différents. En revanche, ils relatent l'histoire d'une idée et sa mise en application avec l'espoir que les leçons apprises se révéleront utiles dans des environnements variés. C'est également pour être portés à l'attention de ceux qui détiennent les pouvoirs de décision qu'ils ont été rédigés et leur montrer que des projets générateurs de revenus, pour et par les femmes, sont viables et jouent un rôle important dans le développement.

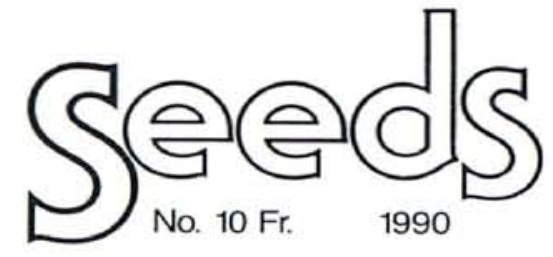

ISSN 073-6833

Copyright (c) 1990 SEEDS
La publication de SEEDS est administrée par le Population Council. Les critères de publication sont déterminés par le Comité de Rédaction de SEEDS: Judith Bruce (The Population Council), Marty Chen (Harvard Institute for International Development), Margaret Clark (The Ford Foundation), Cecilia Lotse (UNICEF), Katharine McKee (Center for Community Self-Help), Anne Walker (International Women's Tribune Center), Mildred Warner (Cornell University), and Ann Leonard (Editor)

Les opinions et commentaires exprimés dans cette brochure sont la seule responsabilité de l'auteur et n'engagent que lui. 


\title{
La Conservation des Forêts au Népal: Encourager la Participation des Femmes
}

\author{
par Augusta Molnar
}

\section{Introduction}

Les femmes des collines du Népal puisent de la forêt les matières premières indispensables à l'économie des foyers: le bois de feu dont elles se servent pour faire la cuisine et chauffer la maison et les feuilles qu'elles utilisent pour nourrir les animaux ou comme engrais. Non seulement obligées de disputer ces produits aux hommes et aux intérêts commerciaux qui coupent les arbres pour en faire du matériau de construction, la situation s'est aggravée en raison du besoin de la population de défricher les forêts pour en faire des cultures agricoles ce qui a résulté en un déboisement massif. L'équilibre délicat des montagnes himalayennes jeunes et instables a été rompu augmentant l'étendue de l'érosion et le nombre de glissements de terrains. Le déboisement est également responsable de l'augmentation du niveau de vase dans le lit des rivières, du détournement du cours des rivières, et de l'inondation dans les plaines du sud. Le déboisement a des effets bouleversants sur la vie des femmes népalaises. Dans le même temps, les femmes, en temps qu'utilisatrices et préservatrices de la forêt, jouent un rôle crucial dans le succès de tout programme visant à sauvegarder la forêt.

Ce numéro de SEEDS porte sur les moyens mis en oeuvre pour faire participer les femmes à un programme gouvernemental visant à préserver et à restituer la forêt. Comme il arrive fréquemment lors de la mise en place de projets aussi vastes ayant une portée générale, les femmes n'étaient pas un centre d'intérêt spécifique au moment de la conception du projet. Cependant, lorsque les activités ont démarré, les responsables du projet népalais et leurs collègues de l'étranger se sont très vite rendus compte que le succès du programme dépendait de la participation directe des femmes aux activités du projet. Au cours des cinq premières années, de 1980 à 1985, on a tenté diverses approches pour mettre en place des activités qui répondaient aux besoins des femmes et suscitaient leur participation. 


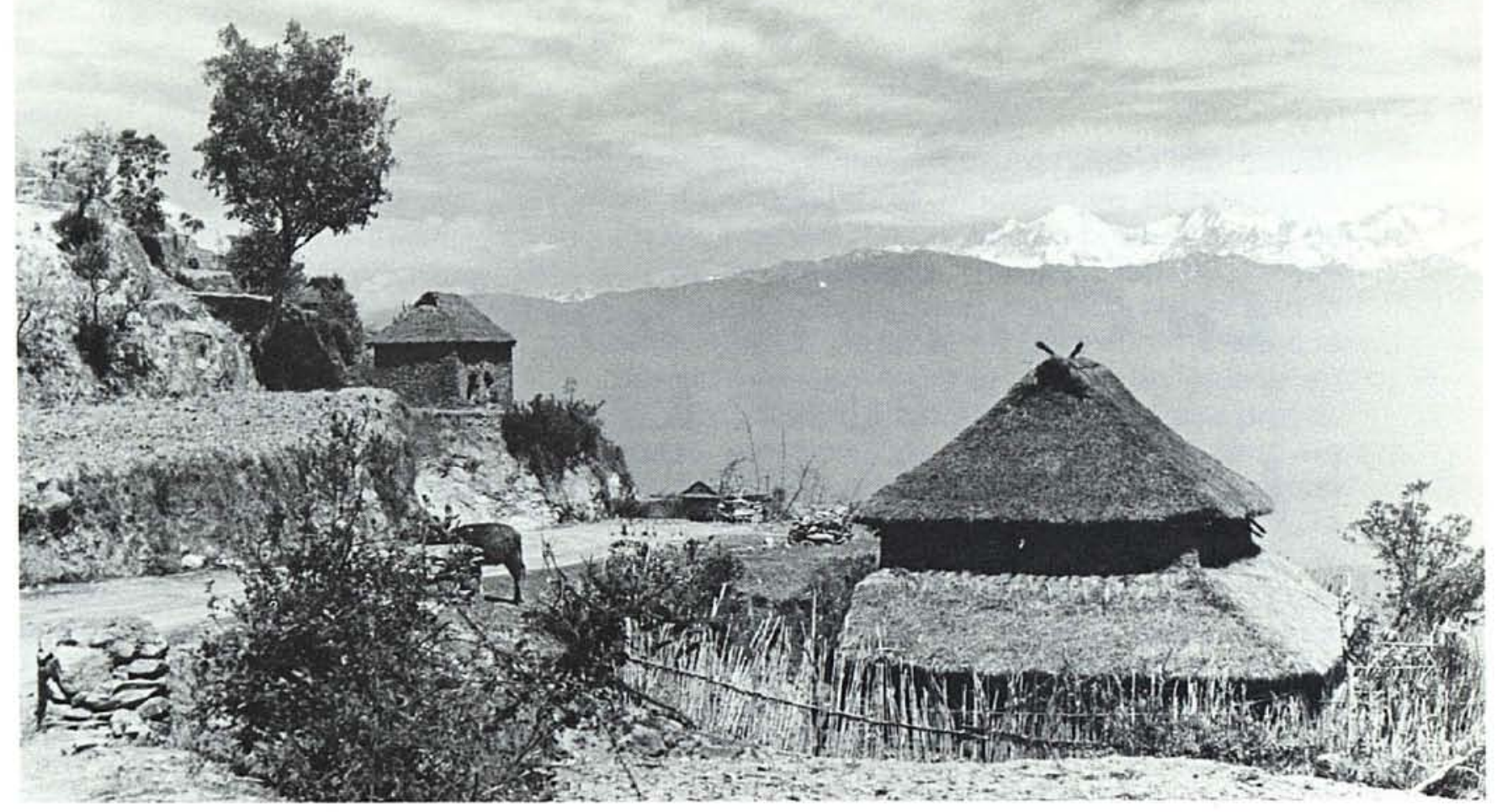

\section{L'Importance de la Forêt dans la Vie dans les Collines Népalaises}

Avec $96 \%$ de sa population demeurant en milieu rural, le Népal est l'un des pays les moins développés au monde. La majorité des ménages habitant en milieu rural vivent d'une économie de subsistance à base d'agriculture et d'élevage. Selon la Banque Mondiale, le revenu par habitant pour l'ensemble du pays s'élevait à US $\$ 140$ en 1980, bien que ce chiffre soit plus bas pour la plupart de la population, ne dépassant pas dans certains cas US \$24 par an. Le taux de mortalité infantile est de 135 pour 1000 naissances vivantes et l'espérance de vie est très basse ( 47 ans pour les hommes et 46 ans pour les femmes). $60 \%$ de la population népalaise demeure dans les régions montagneuses du pays situées au sud de la chaîne himalayenne qui traverse le pays d'est en ouest. Ici, dans les villages parsemés par-ci par-là, les stratégies de subsistance de chaque ménage sont intimement liées aux conditions écologiques variées et complexes.

Bien que le fermier des collines népalais est conscient de l'importance de la forêt pour la protection des sols et des méfaits du déboisement, provoquant des inondations et favorisant l'érosion, le besoin immédiat de disposer de davantage de terres agricoles et de produits forestiers empêche les ménages de contrôler le déboisement à travers leurs pro- pres planification et ressources. Les fermiers népalais ont mis au point un système d'aménagement et de terrassement des terres perfectionné pour limiter la dégradation du sol, mais ce n'est pas suffisant pour stopper l'érosion sur les pentes raides et à faible rendement que l'on cultive de plus en plus.

Le déboisement risque de diminuer les réserves de produits forestiers, notamment, le combustible. $87 \%$ de la consommation en énergie du pays provient du bois et on estime que $95 \%$ de la totalité du bois amassé est utilisé comme combustible. Les besoins en combustible varient selon les différentes collectivités en fonction de l'altitude, du climat, et de l'utilisation de résidus agricoles. On estime que les ménages demeurant dans les basses collines, où le bois est surtout utilisé pour préparer les repas, consomment en moyenne $3,198 \mathrm{~kg}$ de bois par an. Dans les régions plus froides situées à plus haute altitude où il faut également chauffer les maisons, la consommation est encore plus élevée.

A l'heure actuelle, il n'existe pas de substitut au bois de feu utilisé pour faire cuire les aliments qui soit rentable d'un point de vue économique. Dans les plaines du sud, où le bois de feu est encore plus rare, on utilise un mélange de bouses de vaches et de résidus agricoles, une pratique également courante en Inde. L'utilisation d'un tel substitut dans les col- 
lines serait désastreux en raison de l'importance des bouses de vaches dans la production locale d'engrais.

\section{Les Rôles des Femmes dans l'Utilisation de la Forêt}

Les dégâts causés à l'environnement et la pénurie de produits forestiers ont des répercussions sérieuses sur la vie des femmes. Les femmes ainsi que leurs enfants sont géréralement responsables du ramassage du bois de chauffage et d'autres produits forestiers tels que le fourrage pour les animaux et l'engrais végétal. Elles sont également chargées de faire paître le cheptel composé de bovins, d'ovins et de caprins. Le rôle des femmes dans le ramassage de produits forestiers doit être vu dans le contexte de leurs autres tâches ménagères.

Si les femmes sont moins actives que les hommes dans l'économie monétaire (et sont, par conséquent, sous-représentées dans les statistiques nationales sur la participation totale des femmes à la main-d'oeuvre), des études récentes se proposant de calculer le temps alloué aux tâches familiales ont donné des résultats plus réalistes sur la contribution totale des femmes de milieu rural à l'économie des foyers. Une étude menée dans huit villages a révélé que les femmes travaillaient en moyenne 11 heures par jour par rapport à 8 heures pour les hommes. Et si on prend en considération à la fois le revenu total du ménage et la production pour assurer la subsistance du ménage, la contribution des femmes est de 50\% du revenu total, celle des garçons et des filles de $6 \%$ et celle des hommes de $44 \%{ }^{2}$

Cette disparité entre la quantité de travail accompli par les hommes et les femmes est imputable à plusieurs facteurs. Premièrement, en plus de leurs responsabilités en matière d'agriculture et d'élevage, les femmes sont également chargées de préparer les aliments, de ramasser le bois de chauffage et le fourrage, et des corvées ménagères, y compris confectionner les repas et élever les enfants. Deuxièmement, beaucoup d'hommes quittent

\footnotetext{
1. Thompson, M. \& Michael Warburton, "Uncertainty on a Himalayan Scale," Mountain Research and Development. v.5, n.2. pp. 115-135.

Campbell, JG. \& T.N. Bhattarai, "Preliminary Evaluation of Project Effects Results of Key Indicator Surveys," Project Document. Katmandou. Népal 1983, p. 25; et Campbell, J.G. "People and Forests in Hill Nepal," Project Paper Na 10, Katmandou, Népal: 1983

2. Acharya, M. \& L. Bennett. "Women and the Subsistence Sector: Economic Participation and Household Decision Making in Nepal." Washington, DC World Bank Working Paper No. 526, 1985.
}
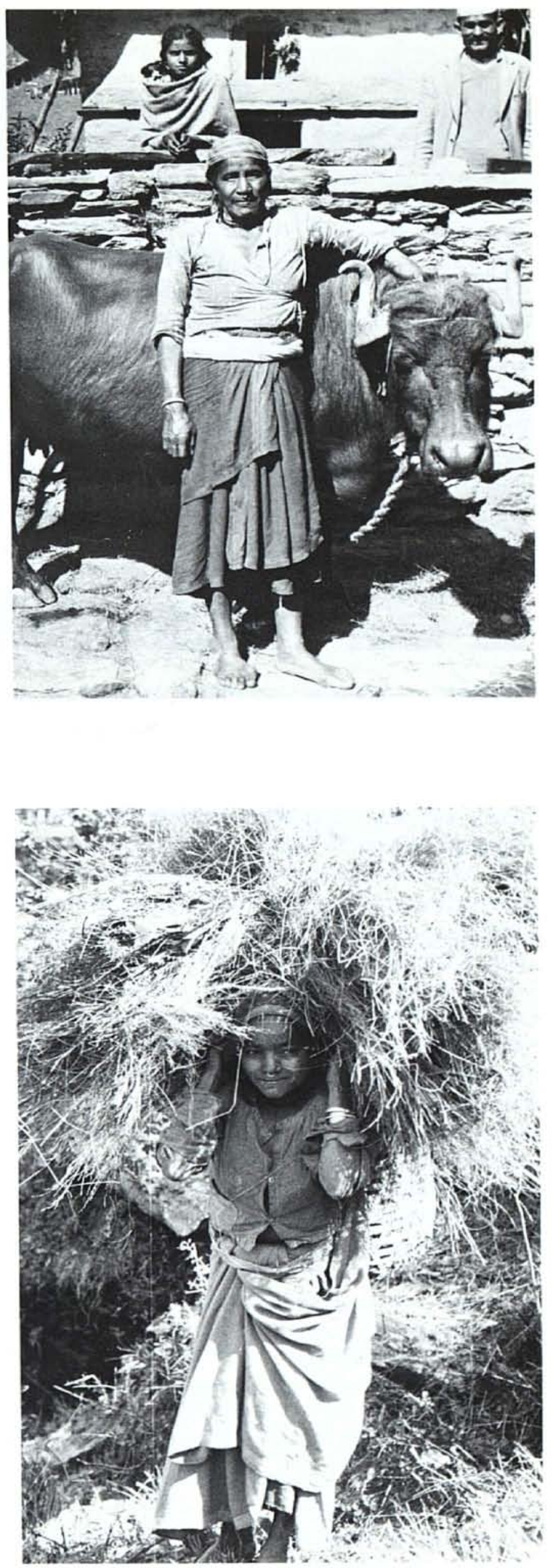


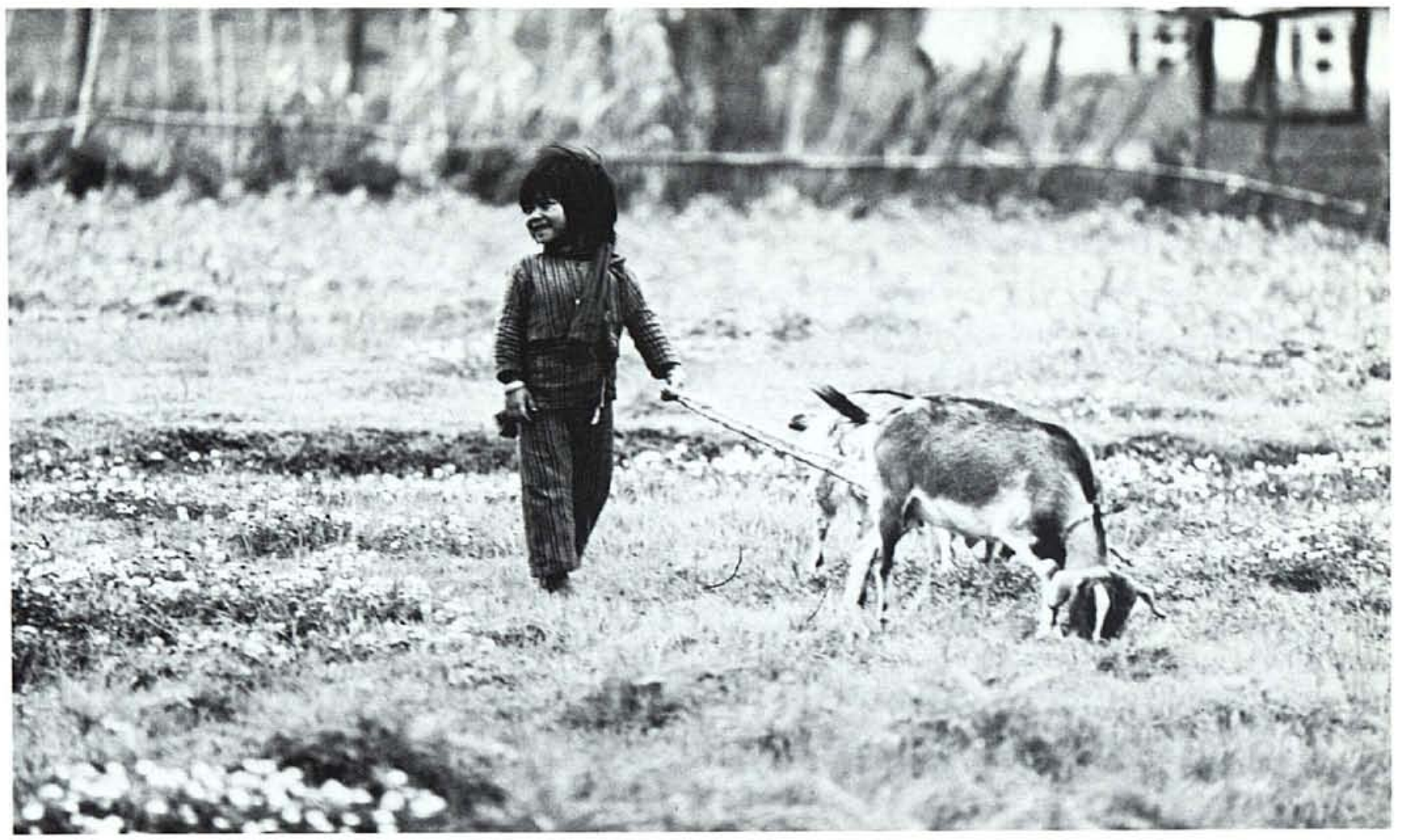

temporairement leur foyer pour s'installer dans d'autres régions du Népal ou en Inde à la recherche d'emplois rémunérés afin de subvenir aux besoins de leur famille. Dans ce cas, les femmes doivent également accomplir une plus grande partie des tâches agricoles.

La diminution des produits forestiers ne fait qu'accroître le fardeau qui pèse sur la femme et sa famille. Krishna Devi, une femme des collines appartenant au groupe ethnique des Magar est contrainte de passer une journée entière à marcher dans la forêt à la recherche de bois de chauffage et de fourrage. Lorsqu'elle était jeune, la forêt adjacente au village dans lequel elle vit abondait en bois de chauffage et en pâturages. A présent, l'augmentation de la population humaine et animale du village a épuisé les ressources, et elle et ses concitoyens doivent parcourir des distances beaucoup plus grandes pour trouver ces produits.

En vertu de la loi, nous, les villageois, avons seulement le droit de ramasser ce qui est tombé au sol de la forêt. Seuls ceux qui ont eu assez de chance pour se faire délivrer un permis par les gardes-forestiers ont le droit de couper des arbres pour en faire du bois de construction. Aux femmes, il ne reste que les feuilles, les branches et les brindilles. II fut un temps où on pouvait encore trouver $d u$ bois mort par-ci, par-là. Mais aujourd'hui ce n'est même pas suffisant pour remplir ma hotte à moins de marcher pendant des kilomètres et des kilomètres. Et pas de fourrage à moins de couper des branches.

Même si je marche longtemps à travers la forêt, je suis tout de même obligée de couper des branches, ce qui est illégal, si je veux en avoir suffisamment pour préparer le repas. Et si un garde-forestier me surprend, soit il confisque mes outils, soit il me dit que je dois payer une amende élevée. Mais ai-je le choix? Vu la situation, il faut que j'emmène ma fille avec moi pour m'aider à ramasser du bois de chauffage et du fourrage et elle est obligée de manquer l'école. Je préférerais qu'elle reçoive une bonne éducation pour lui donner un bon départ dans la vie. Mais je ne peux pas faire autrement. Je suis débordée de travail. En ce moment, je vais à la forêt tous les jours où je ne suis pas occupée à travailler dans les champs ou encore à battre ou moudre le grain. Et une hotte (environ $15 \mathrm{~kg}$ ) suffit à peine pour quelques jours. Si le bois de chauffage deviendra encore plus rare ma fille devra arrêter l'école tout à fait pour m'aider dans mes autres tâches.

Les meilleurs bois sont de plus en plus difficiles à trouver. II faut que je ramasse d'autre espèces qui brûlent très mal. Parfois, une hotte dure à peine deux jours si elle est constituée de bois tendre et de broussailles. Et la fumée 
qui s'en dégage est horrible. Certains bois font picoter mes yeux et donnent au riz un goût amer que mon mari peut à peine supporter.

\section{Le Projet}

Entre 1957 et 1977, après la passation de l'Acte sur la Nationalisation de 1957, toutes les forêts appartenaient au gouvernement népalais et étaient administrées par les responsables du département des forêts qui dépend du Ministère des Forêts. Chaque forêt était placée sous la responsabilité d'un garde-forestier rattaché à un bureau régional des forêts et les droits d'usage étaient extrêmement limités. Les villageois avaient uniquement le droit de ramasser ce qui était tombé au sol et ceux qui voulaient couper du bois pour la construction devaient obtenir un permis du bureau régional des forêts. Cependant, au bout de 20 ans de gestion gouvernementale, l'Acte sur la Nationalisation n'a pas donné les résultats escomptés. Ainsi en 1977, le gouvernement décida d'amender l'Acte sur la Forêt de 1957 en introduisant deux nouvelles lois. La première envisageait le transfert légal de massifs forestiers endommagés aux communes par l'intermédiaire des panchayats ${ }^{3}$, les cellules administratives des communes, pour le reboisement et l'entretien à long-terme. La seconde prévoyait le transfert légal d'un nombre limité de massifs forestiers en bon état aux panchayats adjacents pour être protégés et gérés par les collectivités de la manière qui réponde ${ }^{l}$ ? mieux à leurs besoins.

On formula un nouveau programme forestier en s'appuyant sur cette législation. II s'agissait d'un projet quinquennal ayant pour objectif de tester l'ensemble des stratégies de reboisement et d'utilisation des ressources adaptées aux besoins des collectivités. Ce projet démarra en 1980 et fonctionne à l'heure actuelle dans 29 régions montagneuses du pays. L'assistance technique est offerte par des experts de la Banque Mondiale, du Programme des Nations Unies pour le Développement (PNUD) et de l'Organisation des Nations Unies pour l'Alimentation et l'Agriculture (FAO). Ses objectifs principaux sont de contrôler le déboisement, d'améliorer l'état des forêts et d'accroître les quantités disponibles en bois de

\footnotetext{
3. Panchayat, un terme dAsie du Sud. désigne le gouvernement par un petit groupe de representants élus par le village. Au Népal, le panchayat est une unité de 9 villages ou "tuteurs' qui sont administrés par un groupe de 11 conseillers élus, à la tête duquel siège le Prandhan Panch.
}

chauffage, bois de construction, fourrage et autres produits forestiers pour subvenir aux besoins des habitants. Ce projet est très innovateur en ce sens que les villages sont désormais responsables des ressources de leur forêt et non plus le gouvernement. Les objectifs sont les suivants.

1. Création d'une nouvelle division des forêts au sein du Ministère des Forêts appelée Division des Activités Forestières Communautaires et de Reboisement (DAFC);

2. Développement d'un nouveau programme de formation pour les gardes-forestiers et construction de nouveaux locaux pour tenir les séances de formation;

3. Transfert des forêts aux communes conformément à la nouvelle législation;

4. Reboisement des espaces endommagés confié aux panchayats et création de programmes de gestion communautaire pour toutes les forêts placées sous le contrôle des communes;

5. Mise en place de pépinières collectives pour cultiver les semis en vue de la plantation d'arbres par les communautés et les individus;

6. Recherche sur les espèces à usages multiples et celles donnant du bois de chauffage, du bois de construction et du fourrage qui s'adaptent le mieux au niveau local et dont la croissance est particulièrement rapide; et

7. Distribution de fourneaux à bois de meilleure qualité.

Pour réaliser tous ces objectifs, il a fallu développer un grand nombre d'activités d'encadrement. Les bureaux régionaux des forêts ont formé des agents de terrain (AFCs) pour travailler en collaboration avec les villageois. Les pépinières ont été démarrées et des villageois, sélectionnés par les communes et ayant reçu une formation dans le cadre du projet, en sont responsables. Puisque ce projet était le premier à être touché par la nouvelle législation forestière, il a fallu résoudre toutes les questions administratives et juridiques découlant du transfert des terres. On a également développé des supports éducatifs pour promouvoir toutes les activités du projet. En particulier, un effort de promotion considérable a du être déployé auprès des communes pour convaincre les villageois que les activités prévues ainsi que la nouvelle législation ont été mises en place pour leur bénéfice. 
(En effet, beaucoup de villageois craignent qu'une fois de plus le gouvernement va changer d'avis et nationaliser les nouvelles plantations. Par ailleurs, beaucoup de villageois se méfient des responsables du panchayat, plus particulièrement dans les communautés regroupant des villages de différentes ethnies qui sont administrées par un seul panchayat. Par exemple, un panchayat peut regrouper diverses castes hindoues et un ou plusieurs groupes ethniques d'origine mongolienne. Si le panchayat semble être la formule administrative qui réponde le mieux à la mise en place d'activités au niveau communautaire, sa composition n'est pas toujours représentative de celle de la communauté toute entière.)

On s'est également heurté à une forte opposition de la part des employés du Ministère de la Forêt, car les plus conservateurs d'entre eux craignaient de "perdre le contrôle" sur "leur" forêt et par là leur prestige. II a donc fallu donner des cours de réorientation à l'ensemble du personnel forestier, d'une part, pour les convaincre de la nécessité de placer la gestion des forêts entre les mains des communes et, d'autre part, pour les aider à assumer au mieux leurs nouvelles fonctions en temps que conseillers techniques et gestionnaires plutôt que de devenir des policiers ou des adversaires des villages.

\section{Les Femmes et le Projet Forestier}

Au départ, le document de conception du projet ne faisait aucune référence particulière aux femmes et aucune stratégie n'était envisagée pour les faire participer aux activités prévues à l'exception du programme sur l'utilisation d'un fourneau à bois de meilleure qualité. Les organisateurs partaient du principe que puisque les activités étaient dirigées vers la satisfaction des besoins généraux du ménage, ce projet allait bénéficier pareillement aux deux sexes. Une fois démarré, on s'aperçut très rapidement que pour réussir, il fallait que les femmes prennent une part aussi active que les hommes dans ce projet. Deux facteurs, cependant, allait rendre une telle participation difficile: premièrement, la place que les femmes occupaient traditionnellement dans la société népalaise et, deuxièmement, l'absence de personnel féminin au sein du Département de la Forêt au moment où le projet démarra.
Bien que la société népalaise n'est pas aussi restrictive que certains autres pays d'Asie du Sud, les femmes ne prennent pas une part active dans les affaires publiques ou politiques de leur pays (bien que cette participation varie considérablement selon les différents groupes ethniques très nombreux du Népal). En général, les femmes n'assistent pas aux réunions du panchayat, ni à celles tenues dans le village; elles se déplacent uniquement pour remplir les corvées domestiques et agricoles ou pour rendre visite à leur parents ou ami(e)s. Elles passent très peu de temps dans les lieux publics tels que les maisons de thés omniprésentes que les hommes fréquentent régulièrement pour tenir des discussions informelles. Si les femmes vont dans des endroits publics tels que le marché pour faire leurs emplettes, leur conversation avec des hommes qu'elles ne connaissent pas se limitent aux seuls besoins de la transaction et elles ne restent pas plus longtemps qu'il ne faut. Ceci est particulièrement vrai pour les femmes plus jeunes, mariées ou non.

Pourtant, on s'aperçut très rapidement que les femmes étaient tout aussi concernées que les hommes sinon plus dans l'utilisation des ressources de la forêt, et que la participation active des femmes était indispensable pour remplir certaines tâches, telles que la plantation. En effet, lorsqu'on reboise des massifs forestiers endommagés, ceux-ci doivent être protégés à la fois des hommes et des bêtes pendant plusieurs années pour permettre aux jeunes plants de prendre racine. Les femmes étant généralement celles qui font paître leurs animaux dans la forêt, elles doivent comprendre pourquoi il est nécessaire de protéger certains massifs forestiers. Car si elles ne comprennent ou n'acceptent pas cela, elles auront le sentiment qu'on leur a enlevé des pâturages irremplaçables et s'opposeront à la clôture des forêts. En effet, la clôture des forêts locales oblige les femmes à nourrir leur bétail à l'étable plutôt que de les faire paître ce qui les contraint à passer chaque jour plusieurs heures à couper de l'herbe. Pour éviter ce surcroît de travail, elles seront tentées de faire pénétrer leurs animaux dans les zones protégées en dépit de l'interdiction. C'est la raison pour laquelle, on a passé beaucoup de temps à motiver les femmes afin d'obtenir leur soutien.

Au départ, le personnel du Département de la forêt fut extrêmement surpris de découvrir 


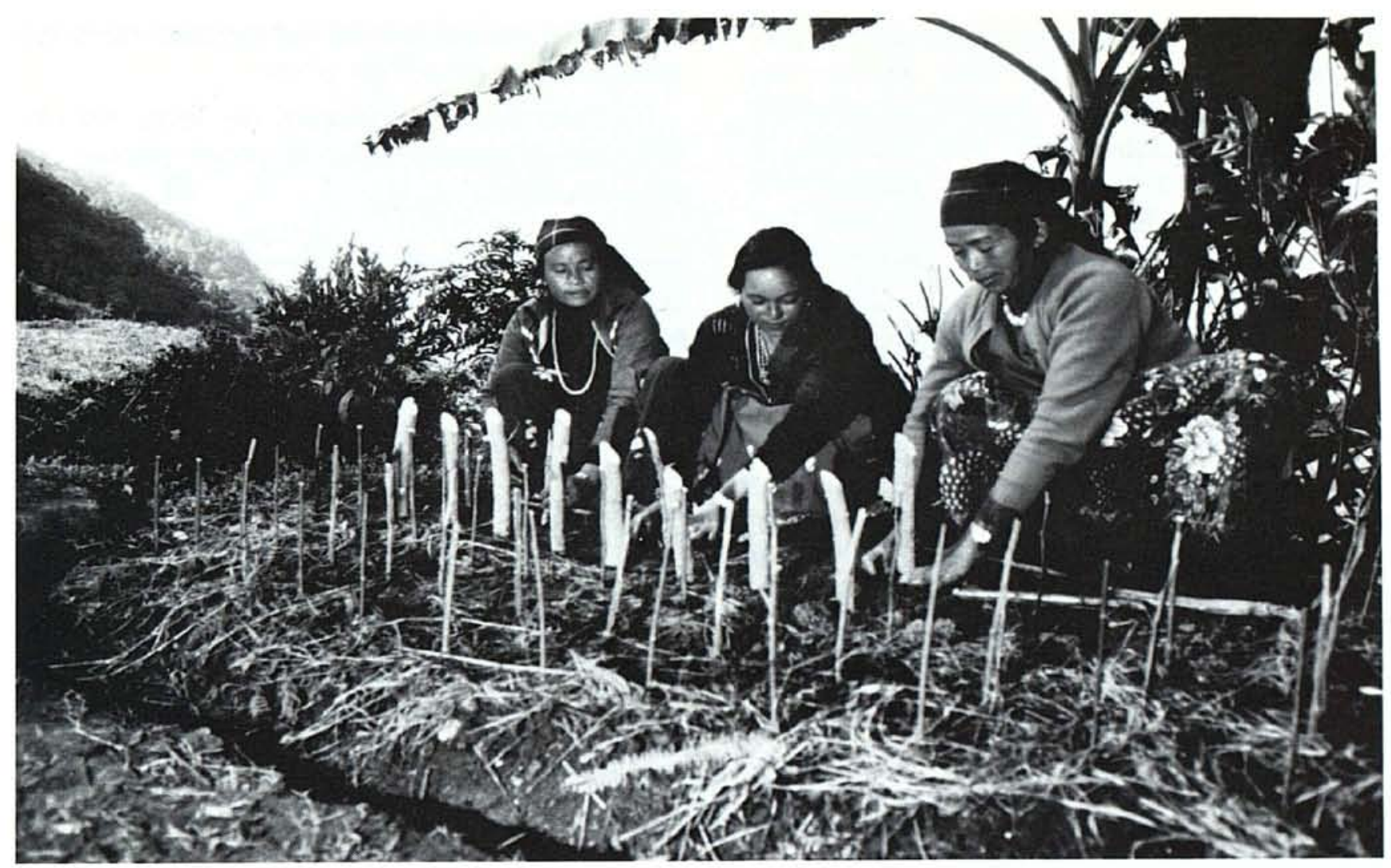

que la plupart des hommes ignoraient quelle était la consommation en bois de chauffage et en fourrage du ménage et s'il y en avait suffisamment dans les forêts environnantes. "Demande à ma femme" fut la réponse généralement donnée par le chef de famille à l'agent de terrain qui lui posait la question.

Les hommes et les femmes ont une préférence pour différentes espèces d'arbres. Comme en témoigne le récit de Krishna Devi, les femmes recherchent des espèces qui possèdent des qualités de combustion spécifiques indépendamment du fait qu'elles poussent rapidement ou s'adaptent facilement au sol et aux conditions climatiques. De même, les hommes et les femmes placent une importance différente sur les produits finis: les hommes sont davantage concernés par la production de bois de construction tandis que les femmes par le fourrage et le bois de chauffage. Le mélange particulier d'arbres donnant du bois de construction, du fourrage ou du bois de chauffage et la quantité d'arbres à couper en une saison pour satisfaire les besoins du ménage sont des questions que les hommes et les femmes abordent de façon totalement différente. II est, par conséquent, tout à fait logique de consulter les femmes si on veut mettre en place un programme de gestion forestière qui fonctionne car si ce programme ne fournit pas suffisamment de fourrage et de bois de chauffage, les femmes vont immanquablement enfreindre les règles.

\section{Faire Participer les Femmes}

L'expérience recueillie dans le cadre du projet a révélé que l'on pouvait éveiller l'intérêt des femmes de trois façons:

1. En analysant les besoins et les rôles joués par les femmes de préférence au moment de la conception du projet et plus tard au moment de sa réalisation pour s'assurer que les femmes en retirent un bénéfice;

2. En canalisant l'information vers les femmes; et

3. En faisant participer activement les femmes soit en les recrutant dans le cadre du projet, au niveau des villages, ou en leur confiant des postes à responsabilité.

Jusqu'à présent, le projet s'est plus particulièrement consacré au déploiement des deux premières stratégies. Si les femmes avaient été un groupe cible dès le départ, on aurait sans doute réussi à recruter un nombre plus important de femmes. Les domaines qui se sont concentrés spécifiquement sur les femmes ont été le développement et la dissémination de matériel d'apprentissage ainsi que la collecte de données sur les progrès accomplis et les problèmes rencontrés par les agents de 
terrain lorsqu'ils ont essayé d'augmenter la participation des femmes. De plus, les femmes ont pris une part active dans le programme consacré à la distribution d'un fourneau à bois de meilleure qualité et font, récemment, partie du personnel de formation et sont des membres actifs dans les comités forestiers des communautés.

Aux difficultés rencontrées pour atteindre les femmes se sont ajoutés des problèmes liés à la complexité et à la diversité des activités du projet ainsi que de son côté innovateur, à la fois en termes d'activités et de mise en place de la structure administrative. L'orientation du personnel du département de la forêt, ainsi que la création de cellules de contrôle et d'évaluation et d'unités de recherche étaient problématiques et ont pris beaucoup de temps et ont, de ce fait, relégué le problème de la participation des femmes au second plan.

\section{Agents de Terrain et Femmes des Villages.}

Recruté dans le cadre du projet, les agents de terrain appelés également AFCs sont rattachés à chaque bureau régional, et ont suivi un programme de formation de deux ans à l'école forestière de Terai. La plupart des recrues viennent de la région de Terai -il y a peu de temps seulement que les habitants des collines ont appris que ces postes étaient à pourvoir. A l'heure actuelle, tous les AFCs sont des hommes parce que l'école n'est pas équipée pour recevoir des femmes. Dans le cadre du projet, on vient de construire une école forestière à Pokhara, une ville située dans les collines, qui accepte des femmes mais l'école n'a pas encore produit de diplômés(es).

Les AFCs ont trouvé qu'il était très difficile d'entrer en contact avec les femmes pour leur prodiguer des conseils techniques. II y a beaucoup de raisons à cela. Premièrement, ils ont été lents à acquérir des techniques de communication efficaces, d'une part, parce que le département n'avait aucune expérience préalable dans ce domaine et, d'autre part, parce qu'il n'y avait pas de matériel éducatif â leur disposition, celui-ci venant à peine d'être développé. Deuxièmement, dans la culture de ces AFCs de la région de Terai, les femmes occupent une place encore plus restrictive dans la société que les femmes des collines. II n'est donc pas surprenant que ces AFCs avaient tendance à éviter les femmes dans les villages où ils étaient en poste.

Ram Kumar, originaire de Terai, est un AFC qui a travaillé pour le projet depuis sa mise en route.

Lorsque j'ai commencé à travailler pour le projet dans les collines, je n'ai jamais adressé la parole aux femmes des villages parce que ce genre de comportement aurait rendu les homme de ma région natale furieux. De plus, les femmes étaient difficiles à trouver; elles n'assistaient jamais aux réunions de groupe avec les hommes et passaient tout leur temps aux corvées ménagères. Nous avons engagé des fémmes pour travailler à la pépinière et s'occuper des plants mais plutôt que de leur donner des conseils je me contentais de les superviser. Au début lorsque je suis arrivé, j'avais du travail par-dessus la tête. La formation que nous avions reçue ne nous avait pas préparé pour faire face à certains problèmes relatifs à la plantation et à la pépinière que j'ai rencontré dans les villages. Certains lopins de terre étaient tellement érodés que c'était un miracle si on réussissait à y faire pousser quoi que ce soit. Quelquefois aussi les paysans avaient plus de connaissances que moi dans certains domaines et j'avais peur qu'ils se moqueraient de mes conseils. J'avais suffisamment de problèmes comme ça sans encore essayer de parler aux femmes.

C'est seulement plus tard lorsqu'on m'a transféré dans un autre district que j'ai réalisé que les femmes jouaient un rôle important dans la réalisation du projet. Le responsable régional pour lequel je travaillais était vraiment exceptionnel et c'est lui qui m'a fait comprendre que les femmes apportaient une perspective différente quant au choix des espèces et à l'utilisation des ressources de la forêt. Nous avons perdu beaucoup de plants parce que les hommes venaient les chercher pour les planter dans leur jardin mais comme les femmes n'étaient pas au courant, les plants étaient piétinés par les enfants ou le bétail.

Lorsque nous nous sommes rendus dans les différents villages pour sélectionner le personnel qui allait faire partie des comités forestiers, déterminer quelles forêts on allait mettre de côté en vue du reboisement ou encore décider quelles stratégies de gestion on allait adopter, on nous avait recommandé de veiller à ce qu'une femme au moins soit élue dans chaque comité. Mais les femmes qui étaient sélectionnées n'intervenaient presque jamais au cours des réunions du comité. Et 


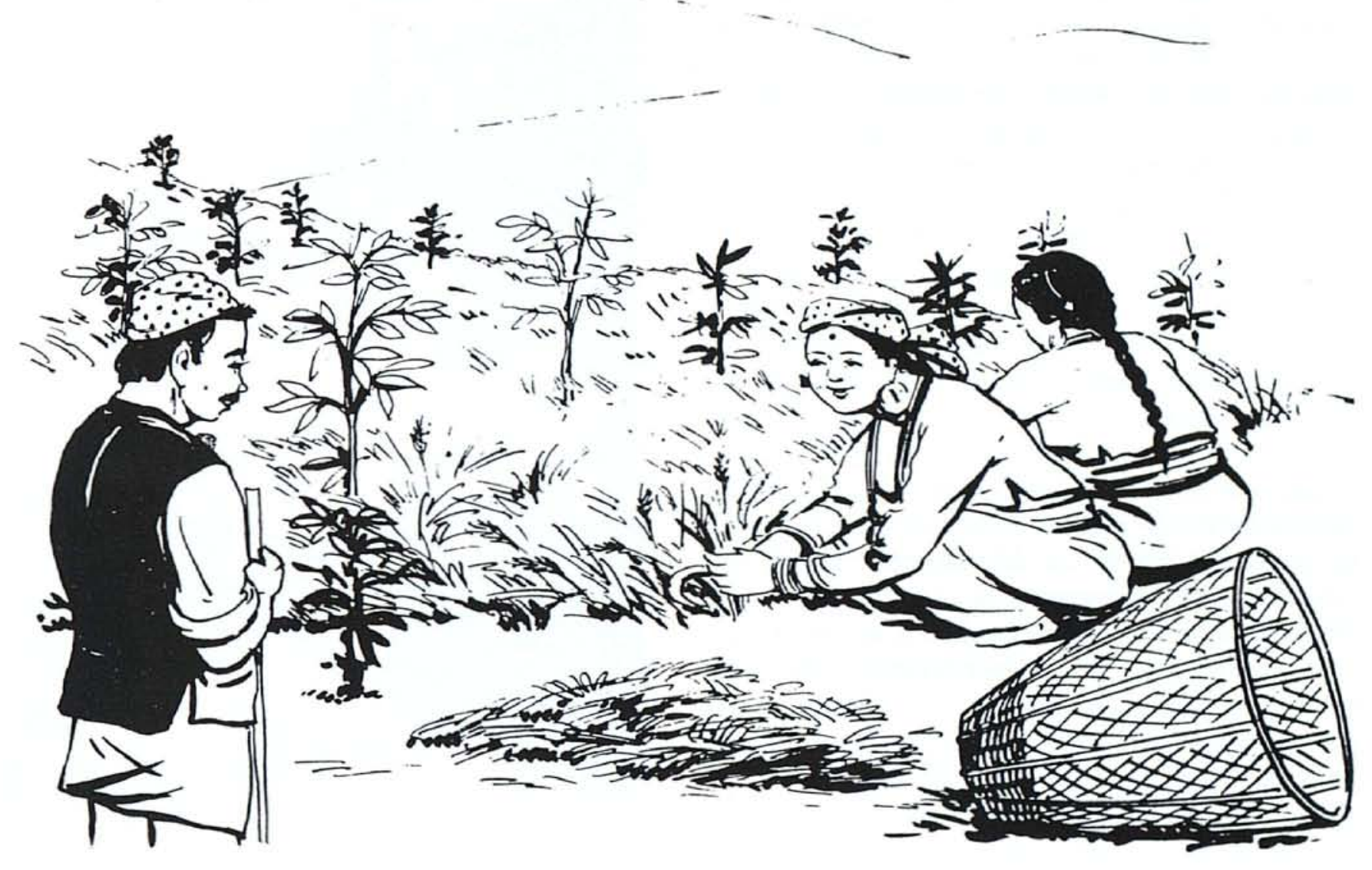

puisque je ne savais pas quelles étaient les femmes influentes ou dynamiques dans les villages, il m'était impossible d'en suggérer d'autres à leur place. Superviser la plantation, délimiter les massifs forestiers, diriger les pépinières et établir le calendrier des activités prenaient tout mon temps. J'étais responsable de quatre villages et ils étaient situés à trois heures de marche l'un de l'autre. J'avais déjà suffisamment à faire avec les hommes sans encore me préoccuper des femmes. Peut-être si on nous avait donné des cours spéciaux durant mes deux années de formation, j'aurais pu faire un meilleur travail.

\section{Participation des Femmes à la Formation et à l'Animation}

Les programmes de formation pour le personnel recruté dans le cadre du projet et au niveau des villages (composé presque exclusivement d'hommes) et le développement de supports éducatifs et leur utilisation par les différentes communes sont des moyens à travers lesquels il est possible de faire participer les femmes des villages et de démontrer le rôle important que jouent les femmes dans la réussite d'un projet forestier. L'ensembe du personnel sur le terrain reçoit une formation-aussi bien les agents de terrain (AFCs) dépendant des bureaux régionaux et couvrant chacun jusqu'à 5 panchayats, que les contremaîtres de pépinière recrutés au niveau des villages, les promotrices de fourneaux, les installateurs de fourneaux, ainsi que les gardes-forêts recrutés localement et qui protègent les forêts nouvellement transférées. Les agents de terrain sont chargés de la formation au niveau des villages.

On a utilisé des supports éducatifs variés, notamment, des brochures informant les communautés sur les droits d'usage des massifs forestiers nouvellement aménagés et ceux venant d'être transférés, des affiches, des montages de diapositives et des dépliants donnant des informations sur les activités déployées dans le cadre du projet (y compris des informations sur les propriétés des différentes espèces d'arbres, sur les techniques de plantation, la nourriture du bétail à l'étable, etc.). Ces supports éducatifs-développés par les responsables du projet en collaboration avec une agence spécialisée dans les techniques de communication élémentaire, le Development Training and Communication Project (DTCP) basé en Thaillande-mettent en lumière le rôle fondamental joué par les femmes dans les 
activités forestières. Ces supports, rédigés en népalais, utilisent un langage simple et de nombreuses illustrations, y compris des dessins de femmes entrain de planter des arbres et s'adonnant à d'autres activités forestières.

Les diapositives et les films projetés au cours des réunions d'information tenues dans les villages ont remporté énormément de succès auprès des villageois. Ces séances auront d'autant plus d'impact si elles ont lieu le soir à l'heure où les femmes, enfin libérées de leurs corvées domestiques, pourront également y assister. Un montage de diapositives intitulé "Le Buffle Doit Manger Tous les Jours" utilise comme caractère principal une villageoise pour démontrer la nécessité de nourrir le bétail à l'étable. La Montagne Fragile ${ }^{4}$, un documentaire consacré à la crise de l'environnement au Népal a été traduit spécialement en népalais pour être projeté au cours de telles séances. Ce film est un support idéal pour faire du travail d'animation avec les femmes parce qu'il leur permet de parler de la manière dont elles perçoivent les problèmes et les solutions. Au cours de ces séances de projection, les femmes généralement bavardent entre elles lorsque le commentateur parle, mais se taisent dès qu'un villageois ou une villageoise est interviewé dans le film. Par ailleurs, un programme consacré en partie à la participation des femmes au projet est également diffusé régulièrement à la radio.

\section{Des Femmes en Temps Que Personnel Forestier}

Dans le cadre du projet, on recrute principalement les femmes pour travailler dans les pépinières, remplir les bacs à semences, arroser les semis et s'occuper de la plantation. Toutefois, ces postes ne contribuent ni à renforcer la participation des femmes dans le processus de prise de décision, ni à pousser les autres femmes des villages à prendre part aux activités du projet. Aujourd'hui, sur les 240 responsables des pépinières, 5 seulement sont des femmes. Dans deux des cas, ces femmes ont repris le poste à la suite du décès de I'homme, un membre de leur famille, qui l'occupait pour prouver que les hommes des villages ne s'opposent aucunement à l'emploi des femmes. Malheureusement la présence de

4. Un film réalisé par Sandra Nichols et diffusé par la chaine de télévision publique américaine dans le cadre de la série NOVA. (Voir Appendices pour plus dinformations)
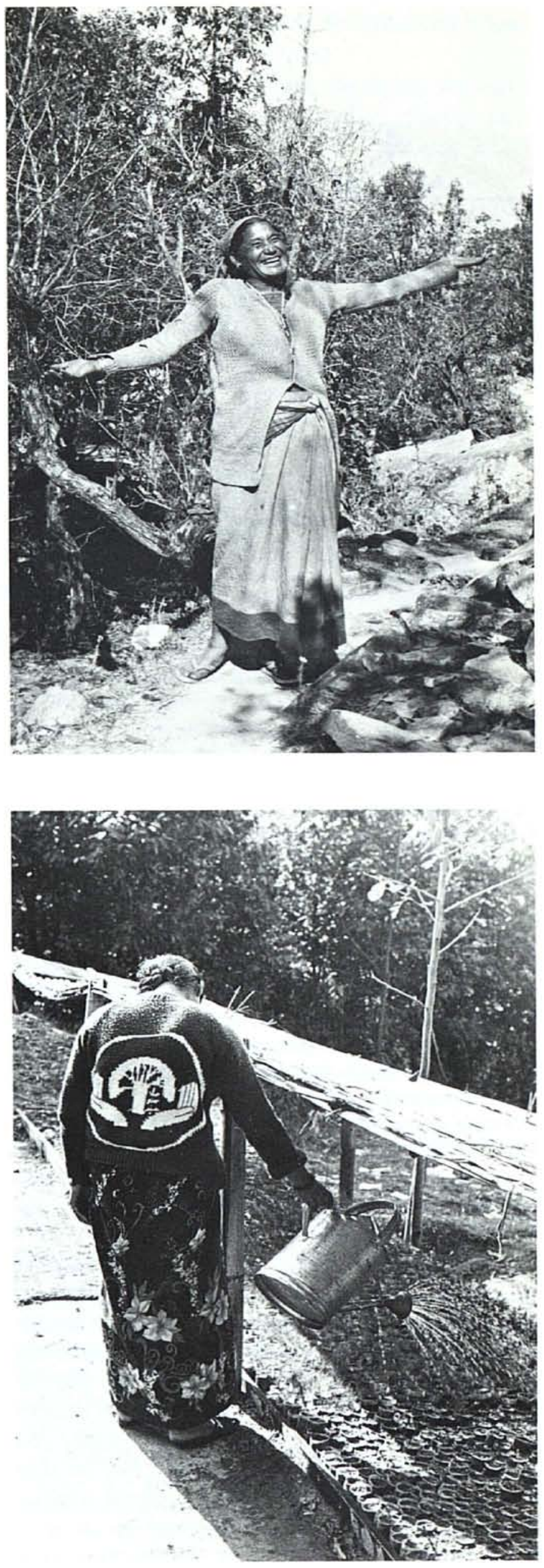
ces femmes n'a pas eu l'impact souhaité en ce sens que cela n'a suffit ni à convaincre d'autres femmes de faire de même, ni à inciter davantage de femmes à venir chercher des plants à la pépinière. En fait, certains travailleurs se sont plaints du manque d'efficacité de ces femmes parce qu'elles ont moins d'autorité que les hommes en raison de leur nature timide et, dans le même temps, ne font aucun effort pour rallier d'autres villageoises. Mais ces problèmes sont sans doute partiellement imputables à la nature accidentelle de leur sélection et auraient pu être éviter si elles avaient été sélectionnées suivant le procédé habituel et avaient bénéficié d'une formation adéquate. Quelques femmes cependant ont été engagées de façon effective en temps que gardes-forêts: des postes réservés à des villageois(es). Les gardes-forêts sont chargés de surveiller les nouvelles plantations et d'éduquer les villageois qui transgressent les lois sur l'utilité du projet. On devrait confier ces postes en priorité à des femmes puisque ce sont généralement des femmes qui vont dans les forêts à la recherche de bois de chauffage et de fourrage.

On a également engagé un certain nombre de travailleurs expatriés, recrutés dans le cadre de programmes de volontaires d'autres pays (Peace Corps, VSOs britanniques, volontaires japonais et programme d'experts associés de la FAO) pour assister les bureaux régionaux et travailler avec les agents de terrain et les villageois. Certains de ces volontaires étaient des femmes et elles ont joué un rôle positif, notamment, en réussissant à identifier certains problèmes et obstacles relatifs au manque de participation des villageoises, en proposant des solutions en vue d'augmenter cette participation et en servant de modèles aux villageois et agents de terrain en démontrant qu'une femme ayant reçu une formation pouvait s'avérer utile dans un projet forestier. Parce qu'elles étaient des étrangères, elles pouvaient se déplacer plus librement sans pour autant susciter la critique des villageois.

II est d'autant plus important de confier des postes de AFC à des femmes puisqu'on prévoit de poursuivre le projet sur une nouvelle période de 5 ans. En ce moment, quelques femmes suivent un cours sur les Ressources Naturelles Renouvelables à l'université de Pokhara. Bien que certaines d'entre elles pourraient être recrutées en temps que AFCs en attendant que les étudiantes de la nouvelle école forestière finissent leurs études, recruter des femmes AFCs tout simplement ne suffit pas pour résoudre tous les problèmes relatifs au statut des femmes et ceux liés au poste d'AFC. En effet, en raison de leur statut social actuel, les femmes AFCs ne seront pas aussi efficaces que leur collègues masculins en travaillant avec les hommes dans les panchayats. Par ailleurs, la présence d'une femme AFC ne signifierait pas nécessairement qu'elle pourrait mobiliser un nombre plus important de femmes si on exigeait d'elle qu'elle accomplisse exactement les mêmes tâches que l'on demande, à l'heure actuelle, aux hommes AFCs d'accomplir. En effet une des plaintes les plus sérieuses des AFCs est qu'ils sont obligés de passer tellement de temps sur la route pour se rendre dans les différents villages qu'il leur reste peu de temps pour parler en tête-à-tête avec les différents fermiers.

II est absolument nécessaire que les responsables du projet trouvent une solution qui permette d'utiliser de façon effective les femmes en temps qu'agent de terrain. Les expériences recueillies dans le cadre de programmes pour les femmes menés au Népal et ailleurs, ont démontré qu'il est nécessaire de mettre en place des programmes de formation spéciaux, d'établir des profils de postes, et de créer des structures de surveillance et d'encadrement. Par exemple, les femmes devront nouer des relations avec les gens dans les différents villages où elles se rendront dans le cadre de leur travail. En effet, on a appris, dans le cadre des programmes de services de planification de la famille, que si des femmes voyageaient seules et étaient contraintes de passer la nuit dans des endroits étrangers, elles faisaient l'objet de médisances rudes. Les femmes AFCs qui ont déjà noué des contacts étroits avec les femmes dans les différents villages dans lesquels elles devront se rendre, et pourront, par conséquent, passer la nuit dans des endroits respectables, sont plus facilement acceptées par la société.

\section{Expérience Avec des Femmes Qui Prennent des Décisions}

Des femmes meneuses ont commencé à émerger au fur et à mesure que les activités se sont étendues sur plus de villages. Au départ, certaines femmes ont émergé spontanément mais il n'y avait pas de moyens formels pour les 
incorporer dans le projet. Un exemple de femme agissant de sa propre initiative est celui d'une habitante de Ilam panchayat, dans l'est du Népal, qui a commencé à s'intéresser aux activités forestières après avoir assisté à la réunion électorale d'une candidate à l'Assemblée Générale. Au cours de cette réunion, à laquelle participait des femmes appartenant à diverses organisations féminines soutenues par le gouvernement, une partie du temps fut consacré à expliquer le nouveau programme forestier. Cette femme fut tellement enthousiasmée par ce qu'elle avait entendu, qu'elle commença à se rendre chez les différents ménages pour leur expliquer les avantages que l'on pouvait retirer en plantant des arbres chez soi et pour encourager les familles à venir chercher des plants à la pépinière. Puisque ses activités de propagande coïncidait avec le début de la saison de plantation, le responsable forestier de la région l'a engagée pour plusieurs mois en la payant avec des fonds provenant du budget pour travailleurs journaliers. A la fin de la saison, il a essayé de l'intégrer de façon plus formelle mais les deux seuls postes rémunérés, contremaître de pépinière et garde-forêts, étaient déjà occupés et il ne disposait pas d'autres fonds.

On peut également citer le cas d'une autre femme toute aussi motivée, appartenant elle aussi à une organisation locale de femmes à Dandeldhura, une région située à l'ouest du pays. Elle avait pris contact avec le bureau régional et les responsables du projet pour obtenir leur soutien afin de pouvoir effectuer du travail d'animation auprès des femmes de la région. Mais le personnel local n'a pas pu l'engager en raison de l'inflexibilité des catégories budgétaires pour les travailleurs recrutés localement. Par ailleurs, elle n'avait aucune envie d'entretenir une pépinière, le seul poste disponible mais voulait se concentrer sur des activités d'animation. Elle espère toujours obtenir de l'aide de la part des employés locaux pour pouvoir organiser des réunions avec les femmes sur la plantation privée et pour obtenir du matériel éducatif. Elle est particulièrement alarmée par le manque de participation des femmes à ce programme et est convaincue qu'un travail d'animation plus intensif qui démontrerait aux femmes les avantages qu'elles peuvent retirer du programme (associé à une meilleure répartition à la pépinière entre les espèces d'arbres donnant des fruits, du fourrage et du combustible) pourrait augmenter de façon considérable le nombre de femmes venant chercher des plants.

Jusqu'à présent, on n'a pas encore trouvé de moyens formels pour intégrer de telles femmes aux activités du projet. Puisque ce projet va servir de modèle pour le lancement d'initiatives similaires à travers l'ensemble du pays, les responsables du projet pensent que la création arbitraire d'un poste unique pour ces femmes exceptionnelles n'est pas une solution viable. Ce qu'il faut c'est mettre en place un système qui permette de recruter ces femmes de manière systématique ou tout au moins permette de les rémunérer. Une solution possible serait d'utiliser les comités forestiers mis en place dans les villages dans le cadre du projet. A deux reprises, des femmes ont fondé de tels comités de leur propre initiative. Dans la région de Kaski, située au coeur du Népal, où, par tradition, les villageois s'occupent euxmêmes de l'entretien des bois, en temps que sous-unités des panchayats, les responsables du projet ont réussi à obtenir une modification de la législation donnant aux sous-unités, plutôt qu'aux panchayats, plus larges et moins homogènes, le pouvoir de contrôler la gestion de leur forêt. Ce comité, établi à ce niveau de sous-unité, fut fondé par une femme intelligente et dynamique qui venait tout juste de finir ses études secondaires et suivait une formation pour devenir enseignante. Elle était partie du principe que la forêt gérée par la communauté répondait particulièrement bien aux besoins des villageois et pensait qu'un comité forestier composé de femmes pouvait être un instrument efficace pour mettre en place des activités sociales.

Un autre comité est composé de 11 membres. Sa fondatrice fut nommée trésorière et la présidente est la femme du chef d'un des villages. Cette femme fut nommée à la tête du comité parce qu'elle était d'âge mur, avait de l'expérience et, grâce à son rang dans la communauté, avait accès à des informations importantes sur les affaires politiques locales. Le comité a déjà supervisé la plantation de 3500 plants dans une région dégradée de leur village et a institué une politique visant à obtenir une somme modeste contre la délivrance d'un permis permettant aux villageois d'utiliser des massifs forestiers existants. Cet argent est destiné à financer des plantations futures et d'améliorer l'infrastructure de la communauté, telle 
que l'installation d'eau courante et la construction d'une nouvelle école.

Un autre aspect intéressant de ces deux comités est qu'ils sont nés de la seule détermination des villageoises bien qu'ils ont, entretemps, obtenu de l'encouragement de la part du personnel de leur bureaux régionaux respectifs. Par ailleurs, les hommes du village ont beaucoup de respect pour ces deux comités et suivent à la lettre les décisions prises par les comités en ce qui concerne l'utilisation des ressources forestières du village. La participation des femmes dans le processus de prise de décisions en matière de gestion des forêts contrôlées par les villages est très certainement une étape cruciale en direction de programmes forestiers à l'échelle communautaire à long-terme.

\section{Fourneaux Sans Fumée Utilisant Moins de Combustible}

Les femmes ont joué un rôle prépondérant dans la partie du projet consacrée à la promotion des nouveaux fourneaux. La majorité des familles népalaises font cuire leur repas sur des fourneaux à bois, le kérosène et le gaz en bouteille étant limité aux régions

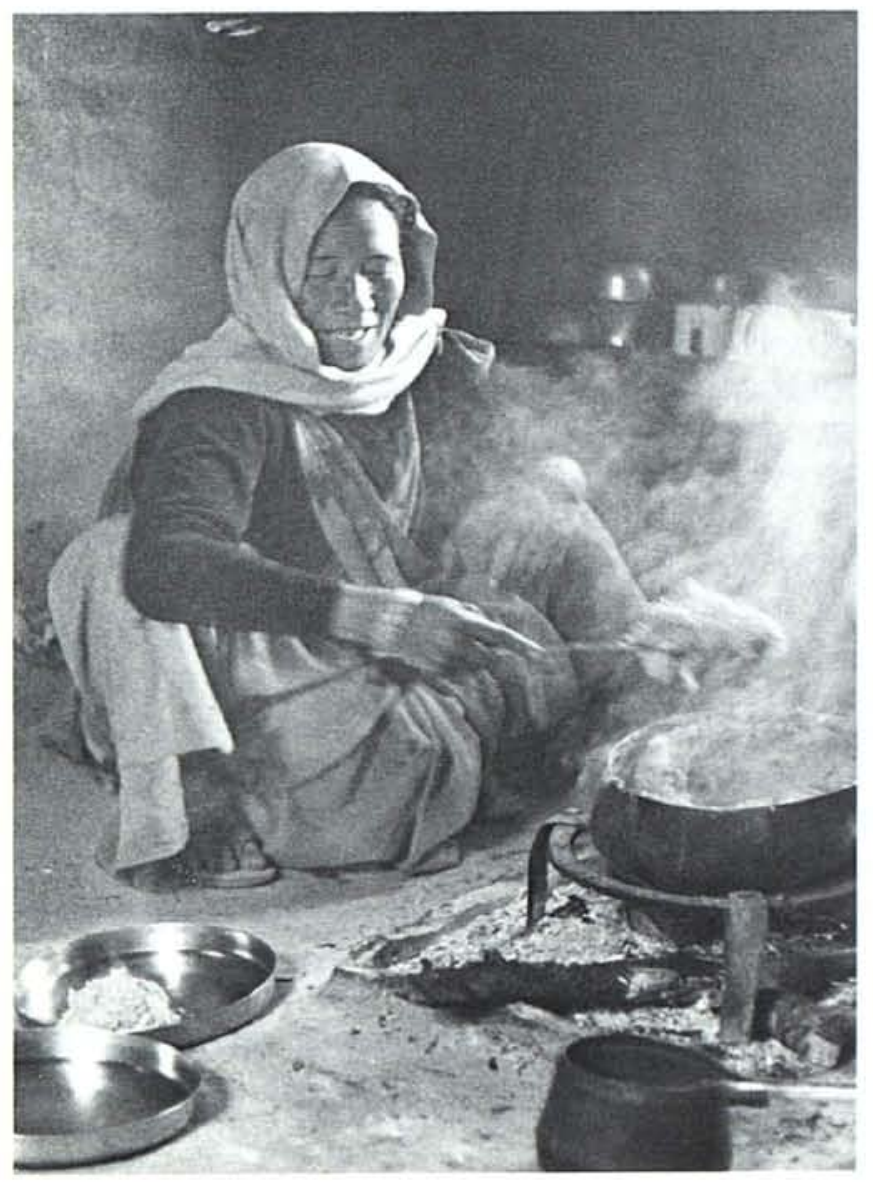

urbaines en raison de leur coût élevé et des difficultés d'approvisionnement. La plupart des femmes et des enfants souffrent d'infections du système respiratoire et de problèmes oculaires en raison de l'exposition à la fumée qui se dégage des fourneaux à bois traditionnels. Des études récentes ont révélé que la quantité de fumée qui se trouvait à l'intérieur d'une maison népalaise était équivalente à celle que l'on produirait en fumant 20 paquets de cigarettes par jour!!

Pour lutter contre ces problèmes de santé et, parallèlement, améliorer la qualité de combustion des fourneaux à bois, la division des forêts, en collaboration avec un institut de recherche népalais et plusieurs groupes internationaux spécialisés dans cette technologie, ont mis au point une série de prototypes similaires à ceux que l'on utilise traditionnellement au Népal. Parmi tous ces modèles, on en a sélectionné un qui était constitué de deux cuvettes en céramique et d'une cheminée en céramique préfabriquée que l'on pouvait monter sur la cage en terre battue. Ce modèle utilisait moins de combustible parce que les pièces préfabriquées étaient construites de telle façon que l'intérieur du fourneau avaient les proportions adéquates et il était bon marché parce que les pièces en céramique pouvaient être fabriquées localement par les potiers népalais traditionnels après qu'ils aient suivi une courte période de formation dispensée dans le cadre du projet. Puisque ces fourneaux étaient montés sur la cage en terre battue traditionnelle, les membres des castes hindoues traditionnelles pouvaient également utiliser ce fourneau puisque les pièces pouvaient être montées sur la structure préexistante: ils auraient rejeter un fourneau fabriqué par des membres appartenant à une caste "inacceptable" s'il venait directement en contact avec leur nourriture. En outre, le fourneau n'est pas muni de soupape pour réguler la circulation d'air (comme c'est le cas pour la plupart des fourneaux modernes en Asie du Sud), il n'est donc pas nécessaire d'apprendre aux femmes à se servir correctement des soupapes de réglage.

On avait envisagé d'installer gratuitement une première série de 15000 fourneaux dans un but promotionnel, dans l'espoir que les familles seraient, par la suite, enclines à acheter les pièces de rechange. Cette première distribution allait également permettre de tester la 


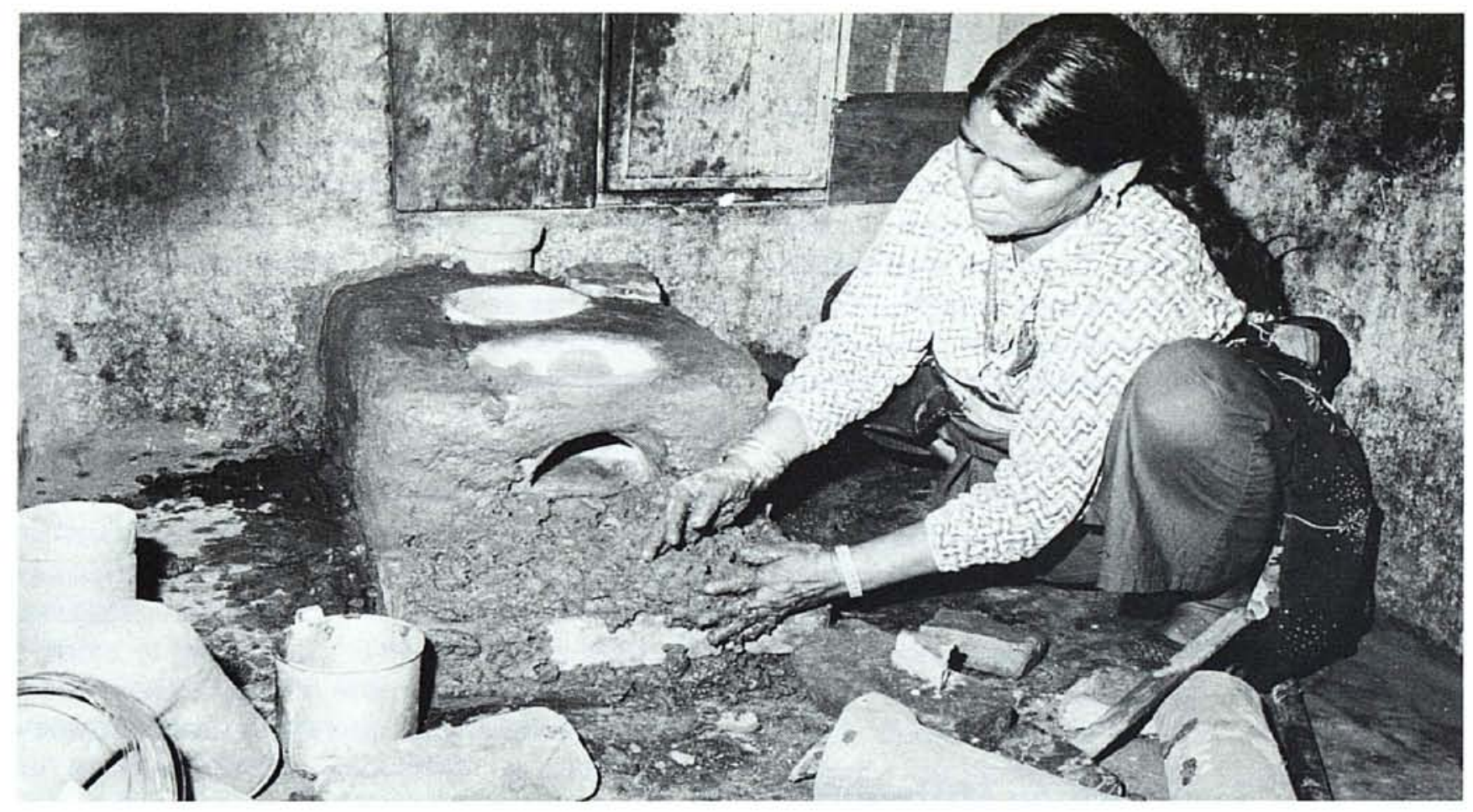

popularité, les techniques de fabrication et le réseau de distribution des fourneaux et de donner une idée sur les stratégies de marketing futures.

Pour distribuer ces fourneaux, on a, dans un premier temps, recruté 9 femmes pour promouvoir les fourneaux et 24 hommes pour les installer avec l'intention de porter ce nombre respectivement à 30 et 60 au fur et à mesure que les régions touchées par la construction des fourneaux allaient augmenter. On a tout d'abord introduit ces fourneaux dans la vallée de Katmandou où les premiers potiers ont été formés parce que le suivi était le plus facile. Par la suite, ce programme s'est étendu sur d'autres régions des collines et les potiers de Katmandou ont formé les potiers dans ces nouvelles régions.

Les premières promotrices étaient de jeunes économes. Elles avaient reçu une formation à la fois dans le domaine de la promotion des fourneaux et dans celui de la gestion des forêts par la communauté pour leur permettre de parler aux femmes aussi bien des activités forestières que de la conservation du combustible. Chandra Devi, une femme de la tribu des Newar, un groupe ethnique de Katmandou, a particulièrement bien réussi en temps que promotrice de fourneaux.

Au début, j'avais beaucoup de mal à éveiller l'intérêt des femmes pour ces nouveaux four- neaux. Elles étaient peu disposées à les essayer. Une chose les intriguait cependant, c'était la cheminée. Une maison népalaise n'est pas très confortable lorsqu'une femme en train de faire la cuisine.

Dans l'ensemble, la réponse à ces fourneaux fut vraiment positive. Bien entendu, nous avons eu pas mal de problèmes mais beaucoup d'entre eux ont pu être résolus. Et les femmes sont prêtes à collaborer avec nous pour trouver des solutions. Au début, la cage en terre battue de certains fourneaux ne contenait pas suffisamment d'argile ce qui a provoqué une fissure de la cage quelques mois seulement après l'installation. Les constructeurs du fourneau ont procédé à des tests supplémentaires et nous avons modifié la composition de la terre battue. Nous avons rencontré le plus de problèmes avec les cheminées. Dans beaucoup de cas, en dépit des spécifications, les installateurs ont été obligés de monter la cheminée plus près du mur extérieur pour éviter qu'elle ne craque. Mais le vent a renvoyé la fumée à l'intérieur de la maison. Plusieurs familles, voulant à tout prix garder leur fourneau, ont fait une installation de fortune en plaçant une boite de kérosène sur le haut de la cheminée pour éviter que le vent ne ramène la fumée à l'intérieur de la maison. II nous faut cependant trouver une solution plus permanente à ce problème.

Si, pour toute une série de raisons, notamment la fissure des cages en terre battue, nous avons moins de gens qu'au cours de la première année, beaucoup de femmes, parcontre, utilisent ces fourneaux exclusivement. 
Nos visites de suivi ont fait toute la différence. Par exemple, nous n'aurions jamais pu détecter toute une série de problèmes et le fait que beaucoup de femmes ont eu du mal à s'adapter à ce nouveau fourneau. Au cours de mes visites, elles ont eu la possibilité de me poser des questions. Parfois, elles se plaignaient que le brûleur avant des fourneaux ne dégageait pas autant de chaleur, alors je leur enseignais différentes techniques pour faire cuire les aliments plus rapidement. Et je peux avoir la conscience tranquille car elles savent comment nettoyer leur cheminée régulièrement. Je leur parle également du programme de plantation d'arbres. Et suite à mes visites, un bon nombre de femmes sont allées chercher des plants à la pépinière.

Jusqu'à présent, ce fourneau semble être bon pour faire des économies de combustible. Une étude réalisée auprès de familles utilisant ce fourneau, a révélé qu'elles économisaient en moyenne $28 \%$ de bois, ce taux atteignant $40 \%$ dans une des régions. Cependant, le taux d'acceptation des fourneaux a diminué pour plusieurs raisons, notamment, la fissure des fourneaux. Des données obtenues en 1983 ont indiqué que $70 \%$ des fourneaux installés étaient utilisés; un an plus tard, ce taux est tombé à $60 \%$.

Puisque la distribution des fourneaux s'étend sur un nombre plus important de régions montagneuses situées hors de Katmandou, il est particulièrement important de continuer les activités de recherche et de développement. Les fourneaux étant toujours distribués gratuitement, il est impossible de déterminer si les familles seront éventuellement prêtes à les acheter. Les régions urbaines, oủ il faut acheter le bois, vont constituer le plus grand marché (on estime qu'un fourneau viable permettra d'économiser de 500 à 800 kg de combustible par an et qu'en trois mois on pourra récupérer la somme dépensée pour acheter le fourneau). Dans les régions rurales, par contre, les économies ne seront pas toujours aussi visibles mais dans les endroits où les femmes doivent parcourir de longues distances pour trouver le combustible, un tel fourneau pourrait finir par devenir une acquisition populaire, bien que dans des proportions plus limitées au départ.

\section{Directions Futures}

Le projet essaye de développer une infrastructure permettant de mettre en place un pro- gramme de gestion forestière qui bénéficiera à tous les népalais, y compris aux femmes. A ce jour, les activités intéressants plus particulièrement les femmes ont été la formation et le développement de matériel éducatif, le système de gestion permettant à la communauté de déterminer comment les ressources locales vont être utilisées, le développement d'un programme de distribution de fourneaux de meilleure qualité, la collecte d'information et de témoignages relatifs aux problèmes et aux approches possibles permettant de faire participer les femmes plus activement en tant que personnel et aux prises de décisions.

Si les femmes sont appelées à prendre une part aussi active que les hommes dans la réalisation du projet, le personnel du projet doit orienter les activités dans ce sens et explorer comment:

-faire participer les femmes plus directement à tous les niveaux et donner aux hommes travaillant dans le cadre du projet les moyens de pouvoir atteindre un nombre plus grand de femmes:

-augmenter la participation des femmes dans les comités forestiers; et

- trouver des moyens permettant de recruter les femmes enthousiasmées par les activités forestières.

Ce sont là des défis importants qui pourraient avoir des répercussions de grande envergure si on intégrait davantage les femmes au projet.

En ce qui concerne la partie du projet consacrée au fourneau, il sera nécessaire, une fois que le fourneau sera distribué sur une plus grande échelle, de se préoccuper de questions telles que le contrôle de qualité des différentes pièces produites en vue de la commercialisation et la mise en place d'un réseau de distribution local adéquat. Cependant, le problème le plus important est de déterminer combien de temps ce programme devrait continuer à être administré par le Département de la Forêt. Beaucoup de gardes-forestiers estiment que ce programme n'a rien à voir avec leur champ d'expertise et qu'il devrait, soit être administré par une autre agence gouvernementale, soit être arrêté une fois que l'on commencera à vendre les fours. D'autres, au contraire, estiment que la conservation du bois est une activité forestière tout aussi importante que la plantation d'arbres et qu'aucune autre agence gouvernementale ne montrera autant d'intérêt dans un tel programme. 


\section{Leçons a Retenir}

1. La clê pour faire participer les femmes dans un projet forestier est de comprendre les rôles centraux joués par les femmes dans l'utilisation des ressources de la forêt et dans son entretien. L'idéal serait d'en tenir compte au moment de la conception du programme. Le personnel doit constamment revoir le projet pour s'assurer que les activités prévues répondent aux besoins des femmes.

2. On devrait adapter les supports éducatifs spécifiquement aux femmes et à leurs intérêts. Ceci peut être réalisé en utilisant des supports que les femmes peuvent comprendre qu'elles soient instruites ou non et en programmant les réunions de telle manière que les femmes pourront y assister. Les supports destinés aux hommes devraient également souligner l'importance de faire participer les femmes au projet.

3. Les femmes soutiendront le projet lorsqu'elles pourront voir des résultats tangibles. Puisque les arbres mettront beaucoup de temps à pousser, des résultats positifs immédiats, tels que l'augmentation de la production d'herbes fourragères ou le stockage dans les pépinières d'espèces d'arbres que les femmes connaissent et apprécient sont des moyens à travers lesquels on obtiendra leur soutien.

4. II est indispensable de faire participer les femmes aux prises de décisions lors de la mise en place de programmes de gestion des forêts par les communautés si on veut que ces programmes soient efficaces. II est important de consulter les femmes au moment de déterminer les variétés d'arbres à planter, le choix des produits forestiers, la quantité à distribuer, quand et à qui, les méthodes de récoltes et la revitalisation des massifs forestiers protégés.

5. Si les employés du projet réalisent qu'il est important de faire participer les femmes plus directement, ils ont également reconnu la complexité du problème que pose une telle participation, notamment dans un domaine aussi vaste que la gestion des forêts par les communautés. Des expériences réussies menées avec des femmes dans d'autres secteurs au Népal et ailleurs en Asie du Sud ont été réalisées sur une petite échelle et dans un espace géographique limité et ont bénéficié d'une planifi- cation locale intensive ainsi que du soutien et de l'encadrement de la part du personnel féminin. Se contenter de créer un "bataillon" de travailleurs femmes pour faire du travail d'animation avec les femmes n'est pas suffisant. II est également nécessaire de mettre en place des structures d'encadrement solides et d'octroyer des ressources suffisantes permettant de recruter du personnel compétent et de surveiller leurs activités.

6. Engager des femmes en temps que personnel forestier permettra d'augmenter la participation des femmes et de mieux répondre à leurs besoins spécifiques. Cependant, à moins d'adapter les tâches exercées par les hommes et les femmes en vue de réaliser ces objectifs, les besoins des femmes ne seront pas forcément identifiés, même si un plus grand nombre de femmes fera partie du personnel.

7. Avant de confier des postes d'agents de terrain à des femmes, il est nécessaire de surmonter les obstacles liés à leur recrutement, leur lieu d'affectation, et leur encadrement. Les problèmes que les agents de terrain femmes rencontreront dans un contexte culturel spécifique doivent être pris en compte au moment d'établir leur profil de poste, lors du recrutement des candidates, en les aidant à s'établir dans les villages dont elles ont la charge, et en mettant en place une structure d'encadrement adéquate.

8. La conservation étant une composante importante pour contrôler la destruction de l'environnement, il est nécessaire que les responsables du projet étudient la manière dont les ressources sont utilisées. Puisque les femmes utilisent une quantité considérable de bois pour faire cuire les aliments, la mise en place d'un programme de distribution de fourneaux de meilleure qualité représente un effort positif en vue de la conservation du combustible qui est complémentaire à la sauvegarde et à la restitution des massifs forestiers. De plus, un tel programme constitue un excellent point de départ pour le personnel du projet pour entrer en contact avec les femmes et parler avec elles de problèmes plus larges touchant à l'environnement.

9. Un programme de distribution en série de fourneaux ne réussira qu'à condition de développer un programme à longterme, qui soit autonome. On ne devrait pas 
greffer un programme de distribution de fourneaux à un projet forestier en espérant que le personnel forestier, devant effectuer d'autres activités, aura suffisamment d'expertise et de temps pour se consacrer à un tel programme. Des mesures pour pouvoir engager du personnel supplémentaire ou pour former et redéfinir les tâches du personnel en place doivent être prises avant de lancer un tel programme sur le terrain.

10. Les agents de terrain femmes se sont révélées être une partie intégrante d'un programme de distribution de fourneaux de meilleure qualité. Au Népal, les femmes ont joué un rôle particulièrement important pour apprendre aux femmes à se servir correctement des nouveaux fourneaux, pour rassembler des informations sur les attitudes des femmes envers les fourneaux et pour informer les femmes sur d'autres aspects de la conservation du bois.
11. La réussite du programme de distribution de fourneaux est, en grande partie, due aux efforts de recherche à long-terme et au développement de modèles qui pourront éventuellement être distribués par le secteur privé. Les expériences recueillies dans le cadre de programmes de distribution de fourneaux de meilleure qualité menés à travers le monde tendent à démontrer qu'il est primordial de (a) développer un produit en gardant à l'esprit les besoins de l'utilisateur final; (b) tester le taux d'acceptation et la rentabilité dans un contexte réel; (c) s'adonner à des activités de recherche et de suivi à long-terme pour corriger les défauts de conception et améliorer la durée de vie du produit; (d) apprendre aux femmes à modifier la façon dont elles font la cuisine et préparent le combustible pour faire une utilisation optimale de leur fourneau; (e) commercialiser les fourneaux en se basant sur les structures de l'économie locale.

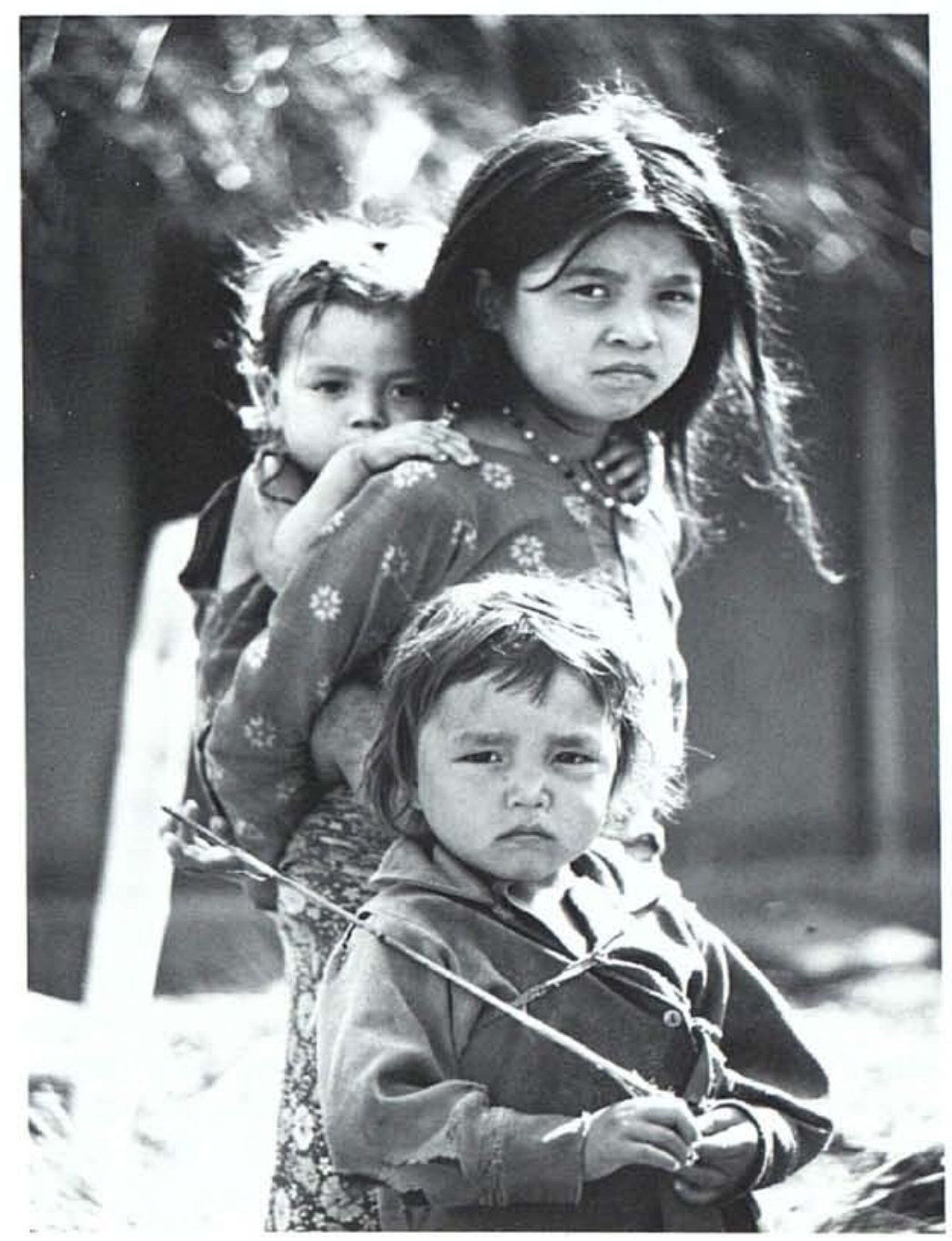




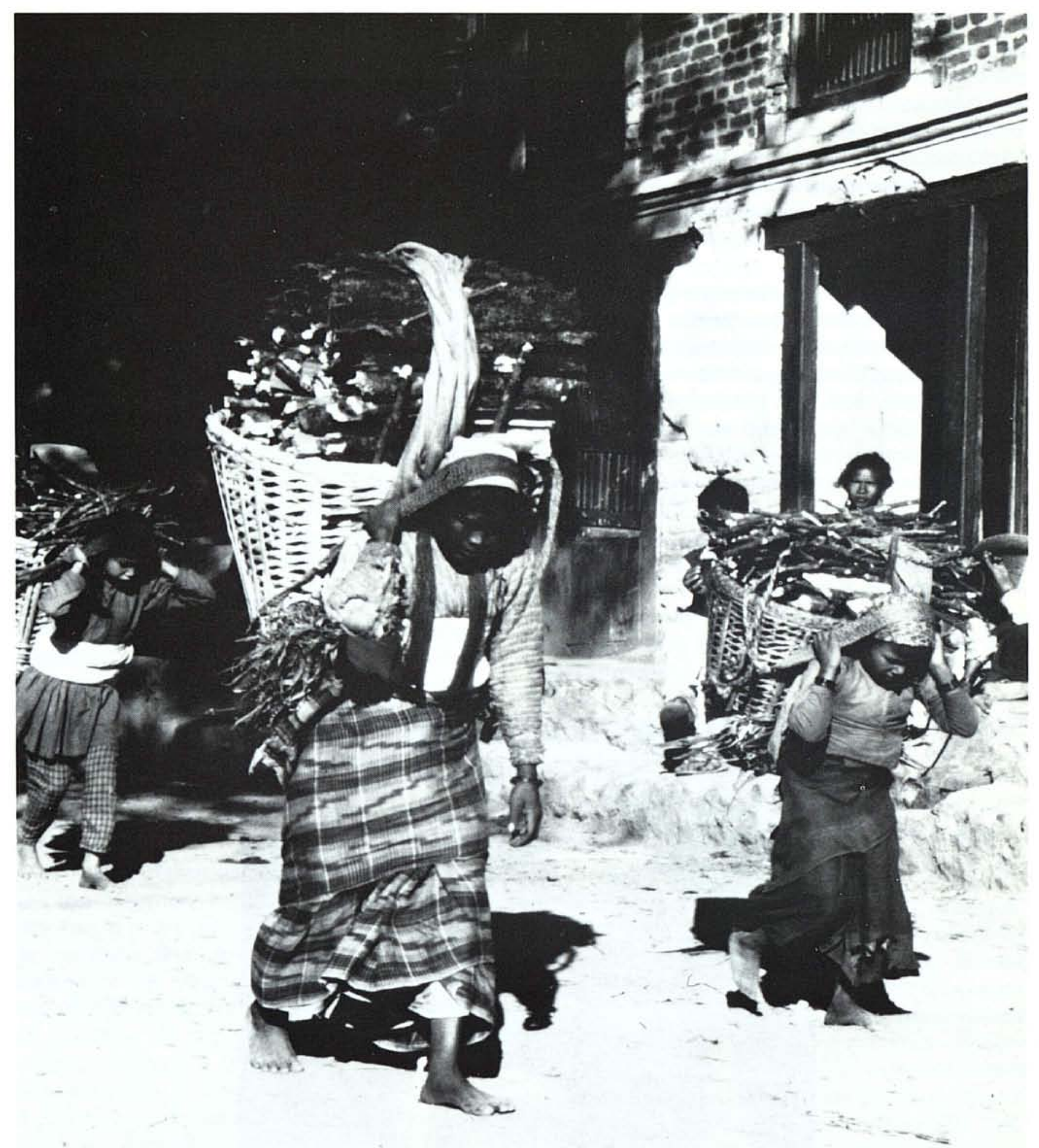




\section{APPENDICE I}

Afrique.

Le résumé, ci-dessous, traite de la participation des femmes à un projet forestier en

\section{Le Projet Agro-forestier de CARE dans la Région de Siaya au Kenya par Hilary Feldstein}

Lancé en 1983 par CARE/Kenya, ce projet, administré par CARE Canada, opère sur des fonds provenant de l'Agence Canadienne de Développement International. L'assistance technique a été fournie par le Centre International de Recherches sur les Activités Agroforestières (International Center for Research in Agroforestry ou ICRAF).

Située dans l'ouest du Kenya, la région de Siaya fut choisi comme région pilote en raison d'une grave pénurie de bois de chauffage. Constituer une réserve de bois de chauffage suffisante et durable en plantant des arbres à croissance rapide et à usages multiples était un des objectifs principaux. Ce projet a été conçu en se basant sur des programmes similaires lancés au Kenya qui avaient démontrés: l'importance de mettre à la disposition des familles des ressources autres que celles provenant de l'agriculture, notamment leur donner accès à des arbres se trouvant sur des propriétés privées; le rôle important joué par les associations de femmes dans la mise en place d'activités communautaires, l'apprentissage de nouvelles technologies, et en temps que source d'information; et les différences entre les hommes et les femmes en ce qui concerne l'accès aux arbres et produits de la forêt et l'utilisation qu'ils en faisaient.

Dès le départ, deux décisions importantes avaient été prises: d'une part, d'utiliser les groupes existants plutôt que de constituer de nouveaux groupes pour travailler dans les pépinières et, d'autre part, de former un nombre égal d'hommes et de femmes aux postes d'agents de terrain chargés de la formation. Le rôle des agents de terrain est de procéder à une première évaluation de la situation avec les groupes en vue de déterminer les pratiques courantes des ces derniers et leur préférence quant au choix des espèces, I'utilisation qui en est faite ainsi que d'autres caractéristiques se rapportant aux arbres avant de mettre en place la pépinière ou de proposer l'utilisation de techniques agro-forestières spécifiques. Le programme veille tout particuliérement à rechercher les opinions et les connaissances des femmes en leur posant des questions sur des sujets qu'elles connaissent parfaitement bien, en s'adressant spécifiquement aux femmes et en parlant avec les femmes soit en temps que groupe ou individuellement mais toujours en dehors de la présence des hommes. On sélectionne les groupes qui disposent d'un site adéquat, qui possèdent un approvisionnement en eau suffisant et qui sont suffisamment motivés pour fournir du personnel volontaire pour s'occuper de la pépinière. On démarre les pépinières durant la courte saison des pluies au mois de Décembre. On distribue l'engrais, les graines et outils nécessaires au groupe qui fournit la maind'oeuvre. En Mars, durant la longue saison des pluies, les plants ont atteint une taille suffisante pour être repiqués. C'est à ce moment que les agents de terrain établissent les plans agroforestiers avec les différents ménages (homme et femme) pour déterminer ce qui est nécessaire à leur exploitation agricole-brise-vent, fourrage, paille, bois de combustible, etc... Les écoles primaires participent également à ce projet et on met particulièrement l'accent sur les différents types d'arbres fruitiers utilisés à l'école et sur les boutures que les enfants peuvent emmener et repiquer chez eux.

Entre 1983 et 1985, le projet s'est étendu de 8 à 225 groupes de femmes et à 75 écoles primaires. Environ 1 million d'arbres ont été plantés au cours de cette période. En 1987. plus de 500 groupes ont déposé des demandes pour prendre part au projet.

On a réussi à atteindre ce niveau d'intérêt élevé de la part des communautés en utilisant une approche basée sur la participation pour déterminer les besoins du groupe, grâce à l'attention minutieuse que les agents de terrain de CARE ont accordée aux individus à tous les stades du projet, depuis l'établissement de la pépinière, à la plantation et au contrôle des opérations, et grâce au fait que l'on a attaché une importance égale aux besoins des hommes et des femmes. Cette approche 
ouverte reposant sur la collaboration eut pour résultat d'augmenter les connaissances sur les espèces locales et d'encourager leur propagation, en mettant l'accent sur des usages multiples du bois autres que le bois de chauffage et le bois d'oeuvre et en donnant des recommen- dations sur la plantation en fonction des besoins agricoles individuels plutôt que d'après un modèle statique.

Pour obtenir plus de renseignements sur ce projet, écrire à: Mme Louise Buck, CARE/ Kenya, P.O. Box 43864, Nairobi, Kenya.

\section{Appendice II}

Ci-dessous, quelques ouvrages de références relatifs à la participation des femmes à des projets forestiers.

\section{Publications}

Agarwal, Anil et Sunita Narain. "Women and Natural Resources." New Delhi: Centre for Science and Environment, 1985.

Agarwal, Bina. "Under the Cooking Pot: The Political Economy of the Domestic Fuel Crisis in Rural South Asia." Sussex, R.U.: Institute of Development Studies, IDS Bulletin, v.18, n.1, 1987.

Foley, Gerald et Patricia Moss. Improved Cooking Stoves in Developing Countries. Londres \& Washington, D.C.: Earthscan, Technical Report No. 2, 1985.

Hoskins, Marilyn. Women in Forestry for Local Community Development: A programming Guide. Washington, D.C.: U.S. Agency for International Development.

Hoskins, Marilyn. Rural Women, Forest Outputs and Forestry Projects. Rome, Italie: FAO Forestry for Local Community Development Programme, 1986.

"Forests, Trees and People," Forestry Topics Report No. 2. Rome: FAO, Via delle Terme di Caracalla, 00100 Rome, Italie. (Disponible également en arabe, en français et en espagnol)

Intermediate Technology Consultants, Ltd. et FAO. Guidelines for the Monitoring of Pilot Stove Development Schemes. Rome, Italie: FAO, Aout, 1985.

Scott, Gloria. "Forestry Projects: How Women Can Help-and Help Themselves." Appropriate Technology, v. 9, n. 3, Décembre, 1982. (IT Publications Ltd., 9 King Street, Londres WC2E $8 \mathrm{HN}$, R.U.)
"Workshop on 'Women's Participation in Forest Management'", Final Recommendations. Katmandou, Népal: Human Resources Development Division, Winrock International, Avril, 1987.

\section{Films}

Nichols, Sandra. La Montagne Fragile. (Disponible en $16 \mathrm{~mm}$, en anglais et en népalais; cassettes vidéo en Betamax ou VHS. Pour obtenir des informations sur l'achat du film, écrivez à: Sandra Nichols Productions, Ltd., 6420 Goldleaf Drive, Bethesda, MD 20817. Pour obtenir des informations sur la location du film, écrivez à: Sandra Nichols Film Library, P.O. Box 315, Franklin Lakes, NJ 07417).

\section{Information sur le Projet}

Pour obtenir des informations sur ce projet et sur l'expérience de la gestion forestière par les communautés, écrivez a:

The Community Forestry and Afforestation Division. Ministry of Forests, Katmandou, Népal (Plusieurs rapports sont disponibles).

Organisation des Nations Unies pour I'Alimentation et I'Agriculture (FAO), Via delle Terme di Caracalla, Rome 00100, Italie.

World Bank. South Asia Projects Division. 1818 H Street, NW., Washington, D.C. 20433. 
Mise en page: Ann Leonard

Typographie: Village Type and Graphics

Photo de Couverture: Nations Unies: J.K. Isaac

Imprimeur: Graphic Impressions, Inc.

Traduction: Pia Heitz

Nous attendons vos remarques, commentaires et idées de projets à publier dans les prochains numéros de SEEDS. Si vous souhaitez recevoir des exemplaires supplémentaires de ce numéro ou faire partie de nos abonnés, n'hésitez pas à nous écrire. Envoyez votre courrier à:

Ann Leonard, éditeur SEEDS

P.O. Box 3923

Grand Central Station

New York, New York 10163 U.S.A. 


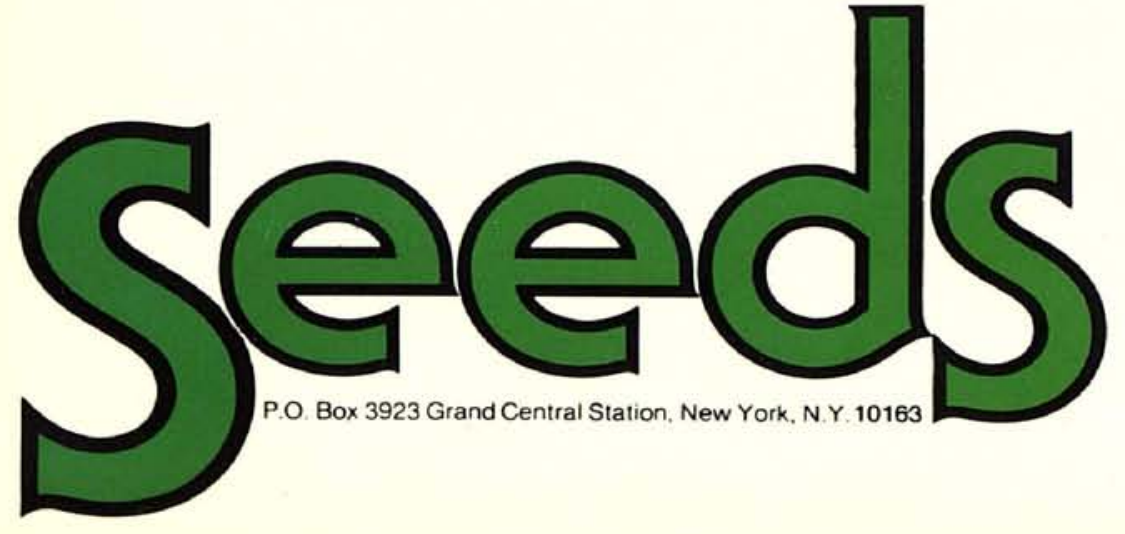

